\title{
Problemas de cooperación en la respuesta a la crisis global
}

\author{
Federico Steinberg \\ Profesor de la Universidad Autónoma de Madrid
}

La cooperación internacional, como el amor pasional, es algo bueno pero difícil de sostener

BenJAMIN COHEN

\section{INTRODUCCIÓN}

La economía mundial se enfrenta a la mayor crisis desde la Gran Depresión. Tras la quiebra del banco de inversión Lehman Brothers en septiembre de 2008, se han producido acontecimientos sin precedentes que han transformado la crisis subprime estadounidense de 2007 en una crisis financiera sistémica con graves consecuencias sobre la economía real.

Los mercados financieros han estado al borde del colapso. Los gobiernos - sobre todo de los países desarrollados - han puesto en marcha planes de rescate del sistema financiero por valor de casi 3 billones de euros que incluyen nacionalizaciones que hasta hace poco eran impensables. La política monetaria ha utilizado fórmulas heterodoxas prohibidas desde hace décadas y además están en marcha paquetes de estímulo fiscal inéditos desde la Segunda Guerra Mundial. El objetivo es conseguir que la recesión en la que están inmersas todas las economías desarrolladas y algunas de las emergentes no se convierta en otra Gran Depresión.

Pero el elevado nivel de interdependencia de la economía mundial, que en parte ha sido el causante de la rápida transmisión de la crisis desde Estados Unidos hacia el resto del mundo, plantea importantes problemas de coordinación a la hora de diseñar las respuestas de política económica. Todos los países se enfrentan a un problema de inconsistencia dinámica por el cual tiene incentivos para romper los acuerdos de cooperación que alcancen - ya sean monetarios, fiscales o comerciales-y adoptar políticas 
unilaterales que les reporten efímeros beneficios a corto plazo pero que corren el riesgo de ser inefectivas a largo plazo y servir sólo para aumentar la deuda pública.

Además, al encontrarnos en un mundo multipolar - y con Estados Unidos, la actual potencia hegemónica, en declive - tampoco existe un liderazgo político claro capaz de resolver dichos problemas de cooperación. El intento del G-20 de erigirse tras su reunión del 15 de noviembre en Washington como el nuevo órgano coordinador de una respuesta global a la crisis se está mostrando inefectivo, lo que aumenta los riesgos de que un nuevo nacionalismo económico haga inviables todos los intentos de adoptar acciones coordinadas que reduzcan el impacto de la crisis y acorten su duración ${ }^{1}$. Incluso dentro de la Unión Europa, donde existen instituciones facilitadotas de la coordinación económica, las respuestas nacionales han estado por encima del esfuerzo colectivo. Mientras algunos países han adoptado políticas fiscales expansivas (ya sea incrementado el gasto público o recortando los impuestos), otros se han resistido a hacerlo, lo que dificulta la recuperación del conjunto de la eurozona por los estrechos vínculos comerciales que existen entre sus Estados Miembros.

Este articulo analiza los problemas de coordinación a los que se enfrentan las principales potencias para dar una respuesta adecuada desde una perspectiva global a la crisis financiera y económica mundial. Tras esbozar brevemente los principales rasgos de la crisis y su impacto en la economía real, analiza desde una perspectiva teórica las dificultades para sostener la cooperación económica internacional y el papel de las instituciones y del liderazgo político en la resolución de dichos dilemas. Además, el artículo explora los pros y los contras de las posibles respuestas coordinadas, con especial énfasis en las acciones de política monetaria y fiscal.

\section{DE LA CRISIS FINANCIERA A LA RECESIÓN ECONÓMICA}

La crisis económica global es el resultado la liberalización financiera de las últimas dos décadas — que no fue acompañada de una nueva regulación adecuada - y del exceso de liquidez global, generado principalmente por Estados Unidos. Ambas alimentaron una euforia financiera que distorsionó la percepción del riesgo, llevando a un exceso de apalancamiento que, sumado al sobreendeudamiento de familias y empresas y a la escasa regulación del sector bancario no tradicional y de los nuevos instrumentos financieros, dieron lugar a burbujas, tanto inmobiliarias como de otros activos. El

${ }^{1}$ La incapacidad de llegar a un acuerdo para cerrar la Ronda de Doha de la OMC antes de fin de 2008 es el caso más claro de las debilidades del G-20. 
desplome de los precios de la vivienda en Estados Unidos precipitó la crisis porque el pinchazo de la burbuja generó importantes pérdidas bancarias, dando lugar a una situación en la que las instituciones financieras se encontraron con demasiada deuda y poco capital. Entonces se vieron obligadas a vender parte de sus títulos (la falta de liquidez les impedía pedir nuevos préstamos a otros bancos), lo que deprimió aún más los precios y generó nuevas pérdidas, además de dejar sin crédito al sector productivo. Este círculo vicioso de desapalancamiento y descapitalización fue a la vez imparable y global. Solo una fuerte intervención pública a finales de septiembre de 2008 pudo frenar el colapso del sistema financiero mundial. Sin embargo, nada pudo evitar una recesión prácticamente global, la peor desde la Segunda Guerra Mundial, que además está teniendo importantes consecuencias geopolíticas.

Lo que en un principio parecía sólo un problema de liquidez se reveló además como un problema de solvencia que está haciendo necesaria una fuerte recapitalización del sistema financiero en los países avanzados, que necesariamente pasa por un rescate del sector público, tanto al sistema bancario como a sectores industriales como el del automóvil. Esto implica un giro radical en los principios liberales que han dominado el modelo de capitalismo anglosajón imperante en prácticamente todo el mundo desde los años noventa, que se está traduciendo en una nacionalización de importantes sectores estratégicos de la economía (Altman, 2008).

Una vez que los paquetes de rescate han alejado el riesgo de quiebra del sistema financiero (de paso redefiniendo el papel de prestamista de última instancia), las principales economías han pasado a activar paquetes de estímulo macroeconómico mediante políticas monetarias y fiscales para intentar acortar la recesión y, especialmente, reducir el aumento del desempleo. Aún así, las principales instituciones internacionales predicen una prolongada recesión en forma de U y el FMI ha revisado al alza su estimación de las pérdidas del sistema bancario mundial derivadas de la crisis financiera. Además, abundan las comparaciones con el crack del 29 y la Gran Depresión.

Así, aunque todavía es posible que aparezcan predicciones más pesimistas en los próximos meses, ya es posible afirmar que todas las economías avanzadas entrarán en recesión en 2009 de forma simultanea, algo inédito desde los años treinta. Según las últimas previsiones de organismos internacionales hechas públicas antes de la publicación de este artículo (Comisión Europea 2009), la producción de la Unión Europea caerá un 1,8\% en 2009, la de Estados Unidos un 1,6\% y la de Japón un 2,4\%. Esta fuerte caída del crecimiento contribuirá a moderar significativamente la inflación, especialmente la de los alimentos, las materias primas y la energía. Además, según el Banco Mundial, el comercio internacional podría caer por primera vez desde 1982, tanto por la falta de crédito y la caída de la demanda como por el aumento del proteccionismo. 
Más allá de esta caída de la actividad es importante señalar las diferencias entre esta crisis y la de los años treinta. En aquella ocasión la economía mundial experimentó deflación, las tasas de desempleo superaron el $20 \%$ en un momento en que los estados no tenían redes de cobertura social como las que existen actualmente y todos los países se lanzaron a las devaluaciones competitivas y al proteccionismo ${ }^{2}$. Además no había economías emergentes (entonces periféricas) capaces de aportar crecimiento y financiación al centro. Por lo tanto, aunque en los próximos años el desempleo crecerá y la inflación caerá es muy probable que la economía mundial pueda escapar de una depresión como la de los años treinta. A ello contribuye de forma sustantiva que la respuestas monetarias y fiscales a la crisis han sido mucho más rápidas y audaces que entonces, demostrando un importante aprendizaje institucional, sobre todo en Estados Unidos.

En cualquier caso, ante la falta de efectividad que está mostrando la política monetaria tradicional, que parece encontrarse en trampa de la liquidez, existe consenso sobre la necesidad de llevar a cabo un gran paquete de estímulo fiscal. Incluso el FMI, tradicionalmente opuesto a este tipo de iniciativas, ha declarado recientemente a través de su Director Gerente que es necesario un estímulo fiscal coordinado del $2 \%$ del PIB mundial (Strauss-Kahn 2008). También ha insistido en que las economías en desarrollo se encontrarán con crecientes problemas para financiar un estímulo fiscal similar al que pueden poner en práctica los países ricos, por lo que necesitarán ayuda extraordinaria de las instituciones financieras multilaterales.

\section{APUNTE TEÓRICO SOBRE LOS PROBLEMAS DE COOPERACIÓN INTERNACIONAL Y LA IMPORTANCIA DEL LIDERAZGO}

Como se ha señalado, la única forma de compensar la pronunciada caída de la demanda global pasa por utilizar las políticas contra--cíclicas y mantener el sistema comercial multilateral abierto. El problema es que para que sea efectiva y reduzca los efectos de la crisis se hace necesario que la respuesta sea coordinada y global, lo que exige elevados niveles de cooperación política internacional. Por ejemplo, si algunos países mantienen políticas comerciales liberales mientras otros elevan el proteccionis-

${ }^{2}$ La aprobación del arancel Smoot-Hawley en Estados Unidos en 1930 inició una escalada del proteccionismo a escala internacional que resultó en una radical contracción de los intercambios internacionales. Para un análisis véase Irwin (2009). 
mo el resultado neto para el conjunto de la economía global no será óptimo. De forma similar, si sólo algunos países realizan un estímulo fiscal, es posible que los aumentos del PIB que obtengan sean limitados y que contribuyan, sin desearlo, a promover la demanda de otros países que no estén estimulando sus economías. En esta sección explicamos brevemente por qué, desde una perspectiva teórica, será difícil alcanzar y sostener la cooperación y evitar las actitudes de free-riding por parte de los estados. Utilizaremos la cooperación en política comercial como ejemplo.

El sistema político y económico internacional es anárquico. No existe una autoridad supranacional capaz de mantener el orden y obligar a los estados a coordinar sus políticas, ni siquiera cuando se han comprometido a hacerlo mediante acuerdos o tratados. Por lo tanto, las relaciones políticas y económicas internacionales se caracterizan por la continua amenaza de conflicto.

A pesar de la anarquía y de la naturaleza no cooperativa del sistema económico internacional, existen multitud de casos en los que es posible alcanzar situaciones en las que varios países salgan beneficiados a través de la coordinación de sus políticas económicas nacionales. La provisión de bienes públicos globales y el mantenimiento del libre comercio son sólo algunos ejemplos. De hecho el creciente proceso de globalización económica ha hecho cada vez más necesaria la cooperación económica internacional porque el aumento de la interdependencia hace que las políticas nacionales tengan efectos cada vez más importantes sobre el conjunto del sistema económico global, lo que requiere de un mayor esfuerzo de coordinación y cooperación si se pretenden maximizar los beneficios de la interdependencia y minimizar sus efectos adversos.

En definitiva, en un mundo económicamente cada vez más integrado, los países, a pesar de tener intereses contrapuestos, están condenados a cooperar. La cooperación económica internacional es la única forma de dar respuestas a los desafíos comunes, como la crisis internacional, y establecer una gobernanza económica global legítima, que se hace cada vez más necesaria (Steinberg 2007).

Sin embargo, los estados tienen incentivos tanto para actuar de forma unilateral (simplemente ignorando las políticas de los demás) como para romper los compromisos adquiridos en el pasado. Es posible modelizar esta estructura de incentivos a través de la teoría de juegos, concretamente mediante los denominados «mixed-motive games», que son juegos caracterizados por una situación de «dependencia mutua y conflicto, asociación y competición» (Schelling 1960:89). El ejemplo más claro es el dilema del prisionero, que describe una situación en la que se produce un problema de acción colectiva en la cual los jugadores son incapaces de alcanzar una situación óptima en términos de Pareto a pesar de que existe una gran convergencia entre sus 
intereses. La estructura de este juego resulta directamente aplicable a la cooperación económica internacional ${ }^{3}$.

Un ejemplo del mismo sería el que aparece en el tabla 1, que muestra la matriz de pagos de un hipotético juego en el que dos países (A y B) tiene la posibilidad de elegir cooperar (practicar el libre comercio) o no cooperar (practicar políticas proteccionistas).

Tabla 1. ¿Libre comercio o proteccionismo?

\begin{tabular}{|c|c|c|c|}
\hline \multicolumn{2}{|c|}{} & \multicolumn{2}{|c|}{ País A } \\
\cline { 3 - 4 } \multicolumn{2}{|c|}{} & Libre comercio & Proteccionismo \\
\hline \multirow{2}{*}{ País B } & Libre comercio & 10,10 & $-10,20$ \\
\cline { 2 - 4 } & Proteccionismo & $20,-10$ & $-5,-5$ \\
\hline
\end{tabular}

Si ambos liberalizan su comercio las ganancias agregadas serán las mayores posibles (casilla superior izquierda). Pero ambos tienen un incentivo para no cooperar porque tienen una estrategia dominante que les indica que, independientemente de lo que haga el otro jugador, su respuesta óptima es el proteccionismo. Así se aseguran que si el otro país protege, ellos no quedarán dañados y si, por alguna razón, el otro país practicara el libre comercio, ellos obtendrían lo que políticamente se percibe como una ventaja (aumentar las exportaciones al otro país manteniendo los mercados interiores cerrados, que viene representado por las casillas superior derecha e inferior izquierda $)^{4}$. El equilibrio del juego, el que resulta de la aplicación de las estrategias dominantes no cooperativas, es el peor en términos de eficiencia paretiana (casilla inferior derecha), donde no es posible que se materialice ninguna de las ganancias del comercio.

Pero la teoría de juegos subraya que no estamos abocados a la no-cooperación. Si el dilema del prisionero se repite un número indeterminado de veces es posible que los países consideren conveniente cooperar porque la estructura de incentivos se ve modificada (Axelrod 1984). Y la cooperación económica internacional, sobre todo la comercial, es un juego que se repite, por lo que no estamos abocados al equilibrio no

${ }^{3}$ Véase Gibbons (1992) para un análisis formal detallado del dilema de prisionero.

4 Téngase en cuenta que la lógica subyacente a la matriz de pagos es la que Krugman (1991:25) llama «mercantilismo iluminado», que poco tiene que ver con las conclusiones de los modelos tradicionales de libre comercio que demuestran que las ganancias del comercio se materializan a través de las importaciones. 
cooperativo, como pone de manifiesto el éxito en las rondas del GATT o incluso el proceso de integración europea.

En definitiva, el dilema del prisionero es un modo de ilustrar los dilemas de acción colectiva que emergen a la hora de proveer un bien público, que en este caso es la existencia de un sistema comercial abierto. En teoría, todos los actores (en este juego son sólo dos pero en la realidad son un gran número de países) tienen incentivos para comportarse como free-riders y no contribuir a su producción, esperando que los demás actores se ocupen de su provisión. Sin embargo, los problemas de acción colectiva son relativamente fáciles de resolver cuando el número de actores es pequeño, ya que en estos casos es posible diseñar mecanismos de control de unos agentes sobre otros (Olson 1965). Dado que muchas de las negociaciones económicas internacionales se producen entre un número relativamente pequeño de Estados, no resulta tan difícil alcanzar la coordinación cuando los intereses subyacentes de los jugadores clave son compatibles ${ }^{5}$. Pero esto no significa que la cooperación sea fácil de sostener a largo plazo.

Por otra parte, la existencia de liderazgo y de instituciones internacionales facilita enormemente la coordinación de políticas. Así, la teoría de la Estabilidad Hegemónica de las relaciones internacionales subraya la necesidad de que una potencia hegemónica actúe como líder para proveer los bienes públicos necesarios para la estabilidad de un sistema económico liberal, abierto y relativamente coordinado (Gilpin 1987, Keohane 1984, Kindleberger 1973) ${ }^{6}$. Si dicho liderazgo se materializa en la creación de insti-

5 Por ejemplo, a pesar de que en las Rondas del GATT participaban un gran número de países, las reducciones de aranceles se negociaban fundamentalmente entre Estados Unidos, la Unión Europea, Japón y Canadá, para posteriormente ser extendidas al resto de naciones a través de la cláusula de la nación más favorecida (Steinberg 2007). Al existir un firme propósito político en avanzar en la liberalización comercial y en mantener un orden económico internacional abierto y liberal, los intereses comerciales básicos de los países industrializados eran compatibles, por lo que fue posible resolver el problema de acción colectiva. Como veremos en la sección siguiente, el establecimiento de regímenes internacionales facilita todavía más la superación de los dilemas de acción colectiva y aumenta los incentivos para la cooperación.

${ }^{6}$ Estos bienes públicos son la provisión de un mercado que absorba los bienes producidos, la generación de un flujo constante de capital, la provisión de un mecanismo que proporcione liquidez al sistema cuando se producen situaciones de crisis de pagos, el manejo de una estructura de tipos de cambio relativamente estables, el establecimiento de una estructura de incentivos para que exista coordinación entre las políticas monetarias nacionales y un sistema básico de definición de los derechos de propiedad para los bienes y el capital que se mueve entre distintos países. 
tuciones o regímenes internacionales con capacidad de establecer sanciones sobre sus miembros que no cumplan los compromisos (como en el caso de la OMC o de la Unión Europea), la matriz de pagos se ve directamente modificada. En ese caso, el cálculo maximizador y egoísta de los estados puede llevarlos a cooperar para no violar las normas de la institución de la que forman parte y así evitar ser sancionados o expulsados. Además, los regímenes internacionales reducen los costes de transacción al establecer redes de comunicación mediante el establecimiento de instituciones formales, lo que reduce los costes de la negociación al permitir a los diplomáticos un rápido acceso a normas previamente aceptadas en materias conflictivas. También aumentan la información, reducen la incertidumbre y permiten organizar y estructurar la interacción repetida entre países al establecer de modo estable unas reglas de comportamiento compartidas, que permiten a los negociadores anticipar el comportamiento de sus colegas de otras nacionalidades. Por último, los regímenes permiten la negociación simultánea de diversos temas interrelacionados, lo que facilita la formación de coaliciones entre distintos países con los mismos intereses, así como el empleo de «pagos compensatorios» que facilitan los acuerdos (Krasner 1983).

Sin embargo, al no existir regímenes institucionales sólidos en las áreas monetaria y fiscal el equilibrio cooperativo no está asegurado ${ }^{7}$. Incluso en materia comercial los acuerdos de la OMC permiten cierto margen de maniobra a sus estados miembros para modificar sus políticas comerciales, lo que supone que algunos pueden ceder parcialmente a la tentación proteccionista sin violar sus compromisos de forma significativa.

Por último, en un mundo multipolar como el actual no existe una potencia hegemónica capaz de tomar las riendas de la situación y aportar en solitario el liderazgo que Estados Unidos aportó tras la Segunda Guerra Mundial. Eso no significa que no pueda haber liderazgo, pero sólo se puede aspirar a un liderazgo compartido que exige reforzar la cooperación multilateral mediante un mejor diálogo entre los países avanzados y las potencias emergentes.

${ }^{7}$ El FMI no puede imponer sanciones a sus estados miembros, sólo puede imponer condicionalidad a aquellos que le han solicitado préstamos. 


\section{RESPUESTAS DE POLÍTICA ECONÓMICA ANTE LA CRISIS}

Una vez analizada brevemente la cooperación en perspectiva teórica, a continuación pasamos a evaluar las posibilidades y dificultades de las respuestas coordinadas a la crisis. Nos referiremos brevemente a la política monetaria y a la fiscal.

En situaciones normales la política monetaria es el principal instrumento que se utiliza para estimular o frenar la actividad económica. Modificar los tipos de interés es más rápido y efectivo que modificar el gasto público o hacer reformas estructurales porque no requiere de pactos políticos ni trámites parlamentarios. Aún así, la ortodoxia en política monetaria requiere que los bancos centrales sean independientes, eviten tener un sesgo inflacionista, se centren en controlar las expectativas de inflación (lo que requiere hacer seguimiento de un gran número de variables) y no moneticen el déficit del sector público ${ }^{8}$.

Pero desde una perspectiva internacional suele resultar difícil coordinar las intervenciones monetarias debido a la existencia de lo que se conoce como el trilema imposible de la macroeconomía abierta, que indica que siempre que exista libre movilidad de capitales los estados tienen que elegir entre mantener una política monetaria autónoma o asegurar la estabilidad cambiaria (Obstfeld y Rogoff 1996, Cohen 1993). Este resultado, que enfatiza que existe un conflicto entre el equilibrio interno y el externo, hace muy difícil alcanzar y sostener la cooperación monetaria al menos por dos razones interrelacionadas. Primero, porque la política monetaria tiene como objetivo principal controlar la inflación interna y los países tienen tanto distintos niveles de inflación como distintos niveles de tolerancia a la misma, por lo que no suelen estar dispuestos a coordinar sus políticas con sus vecinos. Segundo, porque la política monetaria tiene efectos sobre el tipo de cambio, una variable que en ocasiones se utiliza para incentivar las exportaciones y que los estados prefieren utilizar de forma unilateral para lograr este objetivo a menos que se vean obligados a fijar su moneda para ganar credibilidad contra la inflación.

Por lo tanto, los esfuerzos de coordinación monetaria han sido muy limitados desde la ruptura del sistema de tipos de cambio fijos de Bretton Woods en 1971, siendo los más destacables los acuerdos de Bonn (1978), del Plaza (1985) y del

8 Para un análisis de los desarrollos recientes de la teoría monetaria véase Walsh (2003). 
Louvre $(1987)^{9}$. En la actual crisis ha existido cierta coordinación internacional desde agosto de 2007 por parte de los principales bancos centrales del mundo. Por una parte han estado inyectando liquidez en el sistema desde que comenzara la crisis subprime y por otro han bajado los tipos de interés, aunque sólo lo hicieron de forma coordinada el 8 de octubre de 2008, fecha en la redujeron 50 puntos básicos de forma simultánea para que la expansión monetaria no afectara a los tipos de cambio. En el resto de las ocasiones la Reserva Federal y el Banco de Inglaterra se han mostrado mucho más agresivos en sus bajadas que el Banco Central Europeo o el Banco Central Chino (Japón prácticamente no tenía margen porque sus tipos de interés estaban cercanos a cero), y esto ha comenzado a tener efectos sobre los tipos de cambio (sobre todo en el caso de la libra esterlina que se está depreciando con fuerza) y podría tenerlos sobre la inflación, lo que podría dificultar mantener la coordinación en el futuro.

A pesar de estos esfuerzos el principal problema es que la política monetaria está funcionando de forma deficiente. Al encontrarnos ante una crisis de confianza ni las agresivas bajadas de tipos de interés ni las soluciones heterodoxas de expansión cuantitativa de la oferta monetaria que ha puesto en práctica la Reserva Federal están siendo suficientes para devolver la liquidez a los mercados de crédito ${ }^{10}$. Esto se debe a que por primera vez desde la Gran Depresión Estados Unidos y Europa han caído en la trampa de la liquidez (Japón ya cayó en ella en los años noventa y ahora está recayendo). Esta anómala situación se produce cuando el aumento de liquidez no sirve para animar la inversión y el consumo porque las expectativas son tan negativas que tanto empresas como consumidores prefieren no gastar. Para explicarla Keynes decía que la política monetaria es como una cuerda: es efectiva para contraer la economía tirando de ella, pero no siempre funciona para reactivar la actividad porque una cuerda no sirve para empujar, sobre todo si los factores psicológicos que están detrás de las malas expectativas no se modifican.

La situación se vuelve todavía más complicada si la ausencia de gasto privado lleva a una caída del nivel de precios. De hecho, la deflación, en la que afortunadamente la economía mundial todavía no ha caído, es mucho más peligrosa que una inflación

9 Para un análisis de los episodios de cooperación monetaria véase Spero y Hart (2002) y Obstfeld, Shambaugh y Taylor (2005).

${ }^{10}$ Véase Bernanke (2009) para un detallado análisis de los instrumentos que la Reserva Federal ha utilizado para inyectar liquidez al sistema monetario estadounidense e internacional desde el estallido de la crisis, incluyendo aquellos considerados heterodoxos. 
moderada, porque aumenta el coste real de la deuda de familias, empresas y gobiernos, incentiva a posponer el consumo y no permite que haya tipos de interés reales negativos por muy bajos que sean los tipos de intervención del Banco Central. Tanto es así que Rogoff (2008) ha llegado a afirmar que lo único que importa ahora es evitar la deflación y que la solución para salir de la crisis es reducir el endeudamiento empresarial y familiar generando una inflación en el entorno del 6\% que permita «licuar» las deudas. Para lograrlo, ha sugerido que los bancos centrales moneticen directamente un creciente déficit público; es decir, que se olviden de la ortodoxia monetaria ${ }^{11}$.

Aunque hay que continuar haciendo todo lo posible por la vía monetaria, cuando se llega a la trampa de la liquidez es necesaria una expansión fiscal discrecional; es decir, un aumento del gasto público deliberado más allá del que proporcionan los estabilizadores automáticos. Esta política da lugar a un déficit público estructural financiado con deuda que tendrá que ser pagada en el futuro. Esto también supone romper la ortodoxia porque el presupuesto debe estar equilibrado a lo largo del ciclo. Por eso es importante que la inyección de gasto se destine a actividades que permitan aumentar el stock de capital y la productividad de la economía a largo plazo y no a un incremento del gasto corriente. Como las generaciones futuras tendrán que pagar mañana lo que las actuales gasten hoy, lo mínimo exigible es que el gasto permita aumentar el crecimiento potencial a largo plazo. Las infraestructuras y la inversión en I+D o en energías renovables son buenos candidatos para recibir fondos.

Otra alternativa es bajar los impuestos. Sin embargo, resulta más adecuado el aumento del gasto porque si las expectativas de los consumidores son muy negativas, aumentar su renta disponible vía bajada de impuestos no asegura que vayan a aumentar su consumo. También se sabe que el efecto multiplicador del gasto público es mayor que el de la bajada de impuestos porque los ciudadanos tienden a ahorrar parte de su renta disponible mientras que el Estado puede permitirse no hacerlo ${ }^{12}$.

11 Es interesante ver cómo en Estados Unidos la deflación se ve con mucha más preocupación que en la zona euro porque sus secuelas en los años treinta fueron mucho mayores. Esta diferencia en las historias monetarias a ambos lados del Atlántico también explica que, hasta ahora, las bajadas de tipos de interés del Banco Central Europeo hayan sido menos agresivas, así como que todavía no se haya planteado utilizar medidas tan heterodoxas como las de la Reserva Federal.

12 Existe cierto debate teórico sobre si el impacto a largo plazo de una bajada de impuestos podría ser mayor que del aumento del gasto. Para una exposición de los distintos puntos de vista véase Mankiw (2009). 
En cualquier caso, al margen de la forma que tome la expansión fiscal (aumento del gasto, bajada de impuesto o una combinación de ambos) debería ser rápida, temporal, centrada en los grupos más vulnerables de la población y del tamaño adecuado para lograr el impacto deseado. Un exceso de gasto o una excesiva demora en su ejecución pueden volverse contraproducentes porque podrían surtir efecto cuando la economía ya se estuviera recuperando, lo que haría que el impulso fuera pro-cíclico en vez de contra-cíclico y supusiera presiones inflacionistas.

Por último, es importante señalar que aunque el aumento del gasto pueden dar lugar a un aumento de la deuda pública que puede ejercer un efecto expulsión sobre la actividad privada vía mayores tipos de interés, en momentos como el actual en los que la actividad privada está congelada, el riesgo de que esto ocurra es bajo (y el de no estimular la economía es muy elevado).

Más allá de las consideraciones anteriores, que son aplicables a todos los países, es imprescindible que todos los países contribuyan en la medida de lo posible al impulso fiscal para lograr el mayor efecto posible en términos globales. Como parte del gasto nacional sirve para reactivar la demanda externa vía importaciones, si hay países que no aumentan su gasto están aprovechando los fondos públicos de otros de forma oportunista. ${ }^{13}$ Además, desde un punto de vista global, es más eficiente que sean los países con superávit por cuenta corriente, como Alemania, China, Rusia o Arabia Saudita los que aumenten en mayor medida su gasto. Sin embargo, es más probable que vuelva a ser Estados Unidos, que ya tiene enormes desequilibrios públicos y externos, quien lidere la expansión fiscal.

Por el momento, la nueva administración estadounidense ha anunciado un paquete de estimulo fiscal de 800.000 millones de dólares, los países europeos han anunciado un paquete coordinado que asciende a más de 200.000 millones de euros y China invertirá 568.000 millones de dólares en dos años. Otros países como Japón, Corea del Sur o Brasil están diseñando estímulos adicionales, en general menores, lo que demuestra que la inyección de gasto será desigual y puede contribuir a acentuar los desequilibrios macroeconómicos globales en el futuro (mayores déficit gemelos en Estados Unidos y el Reino Unido y superávit en los países emergentes, sobre todo asiáticos).

Por último, a la hora de hablar de cooperación internacional ante la crisis es importante enfatizar la importancia de resistir la tentación proteccionista. Es particularmente

13 Esto posiblemente obligará a relajar la aplicación del Pacto de Estabilidad y Crecimiento de la eurozona, aunque la Comisión haya dicho que continuará vigilando los déficit excesivos. 
importante que los países no eleven sus aranceles e instrumenten devaluaciones competitivas como sucedió durante los años treinta. De hacerlo, estas políticas de empobrecimiento del vecino podrían demorar la recuperación global además de debilitar las perspectivas de que la OMC pueda consolidarse como institución central de la gobernanza económica global (Steinberg 2007).

\section{Conclusión}

La crisis financiera internacional ha devenido en una recesión sin precedentes en todos los países avanzados y en una fuerte desaceleración del crecimiento en las economías emergentes. Ante esta situación se hace necesario un esfuerzo de coordinación de políticas económicas a nivel internacional para evitar que la recesión se traduzca en una prolongada depresión. La cooperación debería centrarse en un esfuerzo de estímulo fiscal global, el mantenimiento del libre comercio y mayores esfuerzos en el área monetaria para salir de la trampa de la liquidez.

En definitiva, se trata de una situación en la que la existencia de intereses compatibles entre los distintos países hace posible coordinar sus políticas nacionales para alcanzar una situación mutuamente beneficiosa a largo plazo. Sin embargo, como hemos mostrado con nuestro análisis de teoría de juegos, la cooperación es difícil de mantener a lo largo del tiempo ya que los estados tienen incentivos para romper los acuerdos cuando pueden obtener mayores beneficios en el corto plazo actuando de forma unilateral. Ejemplos de esta actitud oportunista serían las políticas comerciales y cambiarias de empobrecimiento del vecino puestas en práctica durante los años treinta (y que algunos países han empezado a instrumentar en esta crisis) o la no contribución a un estímulo fiscal global.

Así, cuando el horizonte temporal para los estados es corto debido a los problemas de acción colectiva a los que se enfrentan los gobiernos alcanzar y sostener la cooperación es difícil. La inexistencia de un gobierno global capaz de obligar al cumplimiento de los tratados, que es una característica estructural del sistema político internacional, implica que la cooperación sólo será sostenible cuando exista una continuada convergencia de intereses entre países.

En este contexto se hace especialmente importante un liderazgo político internacional fuerte para facilitar la coordinación. Pero en un mundo un mundo multipolar como el actual no existe una potencia hegemónica capaz de tomar las riendas de la situación. Eso no significa que no pueda haber liderazgo, pero sólo se puede aspirar a un liderazgo compartido. Por lo tanto, las instituciones nacionales de los países avanzados y de 
las potencias emergentes tendrán que coordinarse y además será necesario reforzar los foros de cooperación multilateral, lo que requiere aumentar su legitimidad.

\section{REFERENCIAS BIBLIOGRÁFICAS}

Altman, C. Roger (2008), «The Great Crash, 2008. A Geopolitical Setback for the West», Foreign Affairs, enero/febrero, 2009.

Axelrod, Robert (1984), The evolution of cooperation, Nueva York, Basic Books.

Bernanke, Ben (2009), Policy Responses to the Financial Crisis, London School of Economics Stamp Memorial Lecture, 9 de enero.

Cohen, Benjamin (1993), «The triad and the unholy trinity: problems of international monetary cooperation», en Jeffrey Frieden y David Lake (eds.), International Political Economy, Londres y Nueva York, Bedford, St. Martin's, 2000, págs. 245-256.

Comisión Europea (2009), Interim Forecast, January 2009. Disponible en http://ec.europa.eu/ economy_finance/pdf/2009/interimforecastjanuary/interim_forecast_jan_2009_en.pdf

GiLPIn, Robert (1987), The Political Economy of International Relations, Princeton, NJ., Princeton University Press.

Gibbons, Robert (1992), Game Theory for Applied Economists, Princeton, NJ., Princeton University Press.

Irwin, Douglas (2009), The Battle over Protection: A History of U.S. Trade Policy, de próxima publicación.

Keynes, John Maynard (1936), The general theory of employment, interest, and money, Londres, McMillan and Co. Limited.

Keohane, Robert (1984), After Hegemony: Cooperation and Discord in the World Political Economy, Princeton, NJ., Princeton University Press.

Kindlenberger, Charles (1973), The world in Depression 1929-1939, University of California Press.

KraSner, Stephen (coord.) (1983), International Regimes, Ithaca, Nueva York, Cornell University Press.

Krugman, Paul (1991), «The Move Toward Free Trade Zones», en Policy Implications of Trade and Currency Zones, Simposio organizado por Federal Reserve Bank of Kansas City, Jackson Hole, Wyoming, 22-24 de agosto.

Mankiw N. Gregory (2009), «Is Government Spending Too Easy an Answer?», New York Times, enero.

Obstfeld, Maurice, Shambaugh, Jay C. y Taylor, Alan M. (2005), «The Trilemma in History: Tradeoffs Among Exchange Rates, Monetary Policies, and Capital Mobility», Review of Economics and Statistics. 87, núm. 3, págs. 423-438, agosto. 
ObstFeld, Maurice y Rogoff, Keneth (1996), Foundations of International Macroeconomics, Cambridge, MA., MIT Press.

Olson, Mancur (1965), The Logic of Collective Action: public goods and the theory of groups, Cambridge, Harvard University Press.

Rogoff, Keneth (2008), «Inflation is Now the Lesser Evil», Project Syndicate.

Schelling, Thomas (1960/1980), The Strategy of Conflict, Cambridge, Harvard University Press.

Spero, Joan y Hart, Jeffrey (2002), The Politics of International Economic Relations, Wadsworth, Nueva York.

Steinberg, Federico (2007), Cooperación y conflicto. Comercio internacional en la era de la globalización, Madrid, Akal, 2007.

- (2006), «La economía política del proteccionismo», Cuadernos de Economía, vol. 29, págs. 3-34.

Strauss-Kahn, Dominique (2008), Transcript of a Press Briefing by Dominique Strauss-Kahn, IMF Managing Director, Washington, DC, Saturday, November 15. Disponible en http:// www.imf.org/external/np/tr/2008/tr081115.htm

Walsh, Carl (2003), Monetary Theory and Policy, 2. ${ }^{\text {a }}$ ed., Cambridge, MA., MIT Press.

Wolf, Martin (2008), Fixing Global Finance, Johns Hopkins University Press. 